\title{
An improved license plate characters binarization algorithm based on sub-pixel
}

\author{
Ju Zhi-yong ${ }^{1, \text { a }}$, Wang Chao-nan ${ }^{1, \text { b }}$, He Xiao-lei ${ }^{1, \mathrm{c}}$ \\ ${ }^{1}$ University of Shanghai for science \&Technology, Shanghai 200093, China \\ a juzy@usst.edu.cn, ${ }^{b}$ Chaonanw1012@163.com, c15850695057@163.com
}

Keywords: Binarization; Sub-pixel; Curved surface fitting algorithm; OTSU algorithm.

\begin{abstract}
For the problem of traditional binarization algorithm can't segmentation license plate adhesion or intermittent character effectively. This paper proposes a new binarization algorithm which use a curved surface fitting algorithm to get a new gray level image based on sub-pixel. Experiments show that it could widely combine with binarization algorithm and the algorithm can effectively maintain the topology of the license plate characters, and the edge of license plate characters is very smooth.
\end{abstract}

\section{Introduction}

License plate recognition can be widely used in traffic monitoring and management, license plate anti-theft security, highways management system, parking charge system and so on ${ }^{[1-2]}$. For license plate character segmentation and license plate recognition, license plate image binarization is very important.

In recent years scholars put forward many algorithms. For example, Tang and Fang put forward the algorithm based on blocking clustering ${ }^{[3]}$, this algorithm divided the whole image into many parts, it use each small block grey values to clustering, and then to binarization. This algorithm could be reflected the characteristics of the plate target in binary image and reduce the complexity of the background. But how to divide the whole image is a problem. For example, Li and Song put forward the algorithm based on image histogram ${ }^{[4]}$. This algorithm could be clearly distinguish the original image which is less noise in the image details and the background and detail could be clearly distinguished in license plate image. But for the original image which is motion blur and deformation, this algorithm binarization effect is not good. So this paper proposes a new binarization algorithm based on sub pixel. It can be widely combine with other binarization algorithm and can effectively maintain the topology of the characters, and the edge of characters is very smooth

\section{Image preprocessing}

By the ordinary digital camera to the license plate image, because of the car speed too fast, rainy days or the license plate itself have defiled the captured, most of the license plate image is not very clear. So the license plate image preprocessing for license plate image recognition is very important, and license plate image preprocessing is good or bad will directly affect the effect of the license plate image binarization[5].

\section{Gray level transformation}

Generally taken to license plate image is $3 \mathrm{~d}$ color images. So need to be gray, there are different kinds of methods, as follows:

(1) The maximum value method. This methods set the Red, Green and Blue are equal to the maximum of the three[6].

$\mathrm{R}=\mathrm{G}=B=\max (R, G, B)$

This method can form high brightness grayscale images.

(2)The average method. This methods set the Red, Green and Blue are equal to the average of the three value[7]. 
$R=G=B=\frac{(R+G+B)}{3}$

This method can form a soft gray image.

(3) A weighted average method. This methods set the Red, Green and Blue in different weight and then to average [8].

$$
f(i, j)=0.30 R(i, j)+0.59 G(i, j)+0.11 B(i, j)
$$

According to the human eye sensitivity for different colors, now choose weighted average method .After graying, the effect as shown in the below figure:

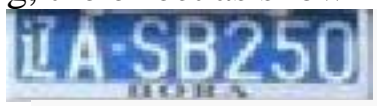

1.1 original image

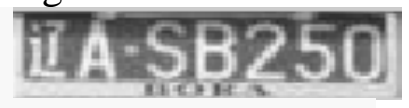

1.2 after graying image

Fig.1 Gray level transformation figure

\section{Improved Algorithm}

\section{Sub pixel division}

This paper set the sub pixel as follow: it divided the original pixel into $n \times n$ parts. (In this paper divided into $3 \times 3$ parts.)Every parts are sub pixels of original pixels. Then magnify the sub pixel as original pixel size, in other words, it magnified the sub pixel $n \times n$ times.

\section{Sub pixel curved surface fitting}

\begin{tabular}{|c|c|c|}
\hline$-1,-1$ & $0,-1$ & $1,-1$ \\
\hline$-1,0$ & 0,0 & 1,0 \\
\hline$-1,1$ & 0,1 & 1,1 \\
\hline
\end{tabular}

Fig. 2 Sub pixels location

Shown as Fig. 2, choose one pixel and around the pixel it choose another 8 points. It assuming that the one pixel coordinates is $(0,0)$, so the other 8 coordinates could know. According the nine gray value of pixel, it could fitting the curve. Setting the curve equation as follow:

$f(x, y)=a_{00}+a_{01} x+a_{10} y+a_{21} x^{2}+a_{12} y^{2}+a_{11} x y, \mathrm{x}$ and $\mathrm{y}$ are the pixel coordinates. $f(x, y)$ is gray value of the pixel. According the gray value of $(0,0)$ and the other four pixels, like $(1,0),(-1,0),(0,1)$, $(0,-1)$,it could get the value of $a_{00}, a_{01}, a_{10}, a_{21}, a_{12}$ :

$$
\begin{aligned}
& a_{00}=f(0,0) \\
& a_{01}=[f(1,0)-f(-1,0)] / 2 \\
& a_{10}=[f(0,1)-f(0,-1)] / 2 \\
& a_{21}=[f(-1,0)+f(1,0)] / 2-f(0,0) \\
& a_{12}=[f(0,-1)+f(0,1)] / 2-f(0,0)
\end{aligned}
$$

After know the 5 value, it could get the rest value of $f(-1,-1), f(-1,1), f(1,-1), f(1,1)$, set as following:

$$
\begin{aligned}
& \varepsilon_{1}=\left[a_{00}-a_{01}+a_{10}+a_{21}+a_{12}-a_{11}-f(-1,1)\right]^{2}=0 \\
& \varepsilon_{2}=\left[a_{00}+a_{01}+a_{10}+a_{21}+a_{12}+a_{11}-f(1,1)\right]^{2}=0 \\
& \varepsilon_{3}=\left[a_{00}-a_{01}-a_{10}+a_{21}+a_{12}+a_{11}-f(-1,-1)\right]^{2}=0 \\
& \varepsilon_{4}=\left[a_{00}+a_{01}-a_{10}+a_{21}+a_{12}-a_{11}-f(1,-1)\right]^{2}=0 \\
& \varepsilon=\varepsilon_{1}+\varepsilon_{2}+\varepsilon_{3}+\varepsilon_{4}
\end{aligned}
$$


Then use $\mathrm{e}^{\frac{d \varepsilon}{d a_{11}}=0}$, it could get:

$a_{11}=[f(1,1)+f(-1,-1)-f(-1,1)-f(1,-1)] / 4$

After all the step, it get all the value of $a_{00}, a_{01}, a_{10}, a_{21}, a_{12}, a_{11}$,so it get the curve equation. If we want to know the gray of sub pixel, it should use the curve equation to integral in each part, then divided this part's area.

\section{Sub pixel by neighboring pixels}

\begin{tabular}{|c|c|c|c|c|}
\hline \multirow{2}{*}{1} & \multicolumn{2}{|c|}{2} & \multirow{2}{*}{3} \\
\hline \multirow{2}{*}{4} & a & b & c & \multirow{2}{*}{} \\
\cline { 2 - 3 } & d & o & f & 6 \\
\cline { 2 - 3 } & g & h & i & \\
\hline \multirow{2}{*}{7} & \multicolumn{2}{|c|}{8} & \multirow{2}{*}{9} \\
\hline
\end{tabular}

Fig. 3 Neighboring pixels

Shown as Fig. 3, choose one pixel and around the pixel it choose another 8 pixel. For it could know which one, use number 1 to 9 to mark and the 9 pixels' gray value it use a to i to mark[9].It set the value of e position equal to the gray value of number $5: f(e)=f(5)$,then with the location of other pixel, could know :

$$
\begin{array}{ll}
f(a)=[f(1)+f(5)] / 2, & f(b)=[f(2)+f(5)] / 2 \\
f(c)=[f(3)+f(5)] / 2, & f(d)=[f(4)+f(5)] / 2 \\
f(f)=[f(6)+f(5)] / 2, & f(g)=[f(7)+f(5)] / 2 \\
f(h)=[f(8)+f(5)] / 2, & f(i)=[f(9)+f(5)] / 2
\end{array}
$$

So use this method also could get the gray value of sub pixel.

\section{OTSU algorithm}

This paper is using otsu algorithm to divide the gray value image into two parts. When the variance of each part reached a maximum value, this gray value is the suitable gray value[10-11]. First divided the whole image into L grayscale, the number of pixel in each grayscale it set as $n_{i}$, the totally number of pixel, it set as $N$. So probability distribution of beach grayscale is $p_{i}=\frac{n_{i}}{N}$.According to the suitable gray value $\mathrm{k}$, it divided the whole pixel into two parts. The first part are the pixels which gray value are between zero and $\mathrm{k}$, the rest is the second part[12].The probability distribution of each part is:

$$
\begin{aligned}
& \omega_{0}=\sum_{i=1}^{k} p_{i}, \omega_{1}=\sum_{i=k+1}^{\mathrm{L}} p_{i} \text {.Seting } \mu(k)=\sum_{i=1}^{k}\left(i \times p_{i}\right), \mu(L)=\sum_{i=1}^{L}\left(i \times p_{i}\right) \text { so it could know } \\
& \mu_{0}=\frac{\sum_{i=1}^{k}\left(i \times p_{i}\right)}{\omega_{0}}=\frac{\mu(k)}{\omega_{0}}, \mu_{1}=\frac{\sum_{i=k+1}^{L}\left(i \times p_{i}\right)}{\omega_{1}}=\frac{[\mu(L)-\mu(k)]}{1-\omega_{0}}
\end{aligned}
$$

Finally get the equation as follow:

$$
\sigma_{B}{ }^{2}=\omega_{0} \omega_{1}\left(\mu_{1}-\mu_{0}\right)^{2}
$$

The suitable gray value $\mathrm{k}$, above-mentioned is the one which could let $\sigma_{\mathrm{B}}{ }^{2}$ have a maximum value.

\section{The experimental results}

The experiment using different kinds of algorithm to binary in license plate image. Comparing the effect of different algorithm, the result shown in below:

Experiment 1: The effect of different algorithm 


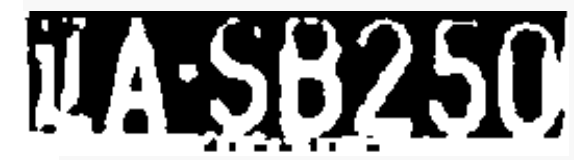

4.1 Ordinary otsu algorithm

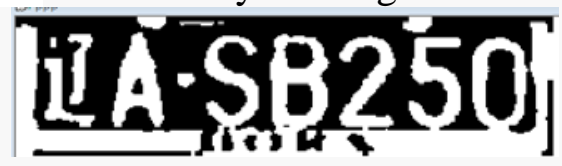

4.3 Sub pixel by neighboring pixels

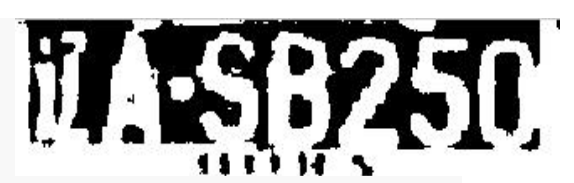

4.2 Histogram algorithm

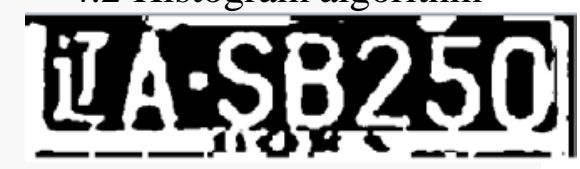

4.4 Sub pixel curved surface fitting

Fig. 4 The effect of different algorithm

This experiment using a fuzzy license plate image to binary. Fig. 4.1 binarization by ordinary otsu algorithm in original image. Fig. 4.2 is binarization by histogram in original image. Fig. 4.3 binarization by otsu algorithm but in sub pixel image which get by neighboring pixels. Figure 4.4 binarization by otsu algorithm but in sub pixel image which get by curved surface fitting. From the figure could know that the edge of font is rough, there are adhesion and broken phenomenon in Fig. 4.1.The edge of font is blur, license plate characteristics is not obvious, character is sticky in Fig. 4.2. The effect of Fig. 4.3 is better than Fig. 4.1 and Fig. 4.2, it can effectively prevent the adhesion and disconnect and the edge of font is smoother. But there are some burrs in Fig. 4.3. Of course could use other way to smooth the burrs, but it will be pay more time. The structure of font keep completely and the edge is smooth in Fig. 4.4. After comparing, conclusion that Fig. 4.4 is the best effect figure.

Experiment 2: The time cost by different kinds of algorithm

Table 1 Time cost by different kinds of algorithm

\begin{tabular}{ccccc}
\hline Algorithm & OTSU & Histogram & Neighboring pixels & Curved surface fitting \\
\hline Time $/ \mathrm{s}$ & $1.1 \mathrm{~s}$ & $1.2 \mathrm{~s}$ & $3.2 \mathrm{~s}$ & $4.1 \mathrm{~s}$ \\
\hline
\end{tabular}

From Table 1 could know that the Neighboring pixels algorithm and Curved surface fitting algorithm take more time to run. Because the two algorithm should get the sub pixel image before binarization. It will take more time, but for the best effect of binarization, this paper choose the Curved surface fitting algorithm. And with the development of technology and science, the processing speed of Curved surface fitting algorithm will be faster and faster.

\section{Conclusion}

This paper put forward an improved algorithm based on sub pixel and the experiments proved that the effect of improved algorithm which should get sub-pixel image before binarization is better. The algorithm improved the effect of license plate image binarization, it could keep the structure of font and the edge is smooth. And the most important is that this sub-pixel algorithm could combine with different kinds of binarization algorithm. It will be widely used in our daily life.

\section{References:}

[1] Wang Guang-yu. The survey on vehicle license plate recognition system [J]. Journal of Zhengzhou Institute of Light Industry: Natural Science, 2001, 16(2):47-50.

[2] Jiang Liang. Analysis of constructing city intelligent transportation system by application of green belt in prefecture-level city[J]. Intelligent City IC,2016(1):80-82.

[3] TANG Quan, FAN Xiao- ping, LI Yan. Binary Method of License Plate Image Based on Blocking - Clustering [J]. Computer Simulation, 2010, 3(27):286-289.

[4] Li Gang, Song Wen-jing. Vehicle License Plate Image Binarization Based on Image Histogram [J]. Journal of Transportation Systems Engineering and Information Technology, 2009, 9(1):113-116.

[5] Rafael C. Gonzalez,Richard E.Woods. Digital Image Processing (Third Edition) [M].Bei Jing: Publishing house of electronics industry, 2012. 
[6] Zhang Zheng, Xu Chao,Ren Shuxia, Han Hailing. Digital Image Processing and Machine Vision( Second Edition) [M].Bei Jing: Posts \& Telecom Press, 2014.

[7] Zhang Liqun, Li Xingfei, Wang Cuo. Gaussian fitting sub-pixel localization algorithm based on sliding edge points [J]. Computer Applications and Software, 2016(1):190-194.

[8] Zhang Shuai, Pei Cai-yan,Zhao Wei,Liu Ai-yong. A Polishing Algorithm of Edge Detection [J]. Journal of Hebei University of Technology, 2010, 39(2):52-55.

[9] Duaa H. AlSaeed, Ahmed Bouridane, Ali ElZaart, Rachid Sammouda. Two modified Otsu image segmentation methods based on Lognormal and Gamma distribution models[C]. Information Technology and e-Services (ICITeS), 2012 International Conference on. Tunisia: Sousse, IEEE, 2012(3):1-5.

[10] Q. Chen, L. Zhao,J. Lu, G. Kuang. Modified two-dimensional otsu image segmentation algorithm and fast realization [J]. IET Image Processing, 2012(6), 426-433.

[11] Shen Xuanjing,Pan Hong,Chen Haipeng. Medical Image Segmentation Algorithm Based on One-Dimensional Otsu Multiple Threshold[J]. Journal of Jilin University: Sci Ed,2016(2):344-348.

[12] HAN Yonghua, WANG Yaming, SUN Qi,ZHAO Yun. Crop Row Detection Based on Wavelet Transformation and Otsu Segmentation Algorithm[J]. Journal of Electronics \& Information Technology,2016(1):63-70. 